\title{
Selective enrichment and metagenomic analysis of three novel comammox Nitrospira in a urine-fed membrane bioreactor
}

\author{
Jiyun $\mathrm{Li}^{1,5}$, Zheng-Shuang Hua (iD ${ }^{2,5}$, Tao Liu (iD ${ }^{3}$, Chengwen Wang $\mathbb{D}^{1 凶}{ }^{凶}$, Jie $\mathrm{Li}^{3}, \mathrm{Ge} \mathrm{Bai}^{1}$, Sebastian Lücker (iD ${ }^{4}$, Mike S. M. Jetten ${ }^{4}$, \\ Min Zheng $\mathbb{D}^{3 凶}$ and Jianhua Guo (iD ${ }^{3 凶}$
}

(c) The Author(s) 2021

The discovery of complete ammonia-oxidizing (comammox) Nitrospira has added an important new process to the microbial nitrogen cycle. While comammox Nitrospira have been detected in various ecosystems, only few studies have achieved their enrichment over other canonical nitrifiers. Here, we obtained a selective enrichment of comammox Nitrospira in a urine-fed membrane bioreactor in less than 200 days. By using 16S rRNA gene amplicon sequencing and quantitative PCR of the functional marker gene $a m o A$, we observed a dominance (up to 30\% relative abundance) of comammox Nitrospira over ammonia-oxidizing bacteria and archaea. Furthermore, the complete genomes of three new clade A comammox Nitrospira were recovered by metagenomics. These three strains were divergent from previously reported comammox species according to comparative genome and amoA-based analyses. In addition to the key genes for ammonia and nitrite oxidation, the three recovered genomes contained a complete urea utilization pathway. Our findings suggest that the urea present in the urine media played a significant role in the selective enrichment of these novel comammox Nitrospira, and support the diversity and versatility of their metabolism.

ISME Communications (2021)1:7; https://doi.org/10.1038/s43705-021-00005-3

\section{INTRODUCTION}

Nitrification, an essential part of the global microbial nitrogen cycle, was for a long time assumed to be a two-step process catalyzed by distinct guilds of chemolithoautotrophic microorganisms. ${ }^{1}$ These included ammonia-oxidizing bacteria (AOB) or archaea $(A O A)^{2,3}$ and nitrite-oxidizing bacteria (NOB). The existence of complete ammonia oxidizers was discussed in an excellent theoretical paper in 2006, ${ }^{4}$ and in 2015 two experimental studies independently documented the enrichment and characterization of complete ammonia-oxidizing (comammox) Nitrospira. ${ }^{5,6}$ Intriguingly, the enriched comammox species seemed to belong to Nitrospira sublineage II, which was previously regarded as a canonical NOB-affiliated group. ${ }^{7}$ Based on the phylogenetic analysis of the gene encoding subunit $A$ of ammonia monooxygenase $(a m o A)$, comammox Nitrospira forms two divergent sister clades, namely the comammox Nitrospira clades A and B. ${ }^{6}$ Moreover, kinetics of comammox Nitrospira determined with the only available pure culture revealed a high affinity for ammonia, the low maximum rate of ammonia oxidation, and high growth yield compared to canonical nitrifiers. ${ }^{8}$ These kinetics were recently corroborated in a highly enriched culture that was dominated by a novel comammox Nitrospira, which surprisingly also was partly inhibited by very low ammonium concentrations $(>25 \mu \mathrm{M})$ in the media. ${ }^{9}$ These findings imply an adaptation to oligotrophic conditions and suggest that comammox Nitrospira can play a key role in nitrification especially in substrate-limited environments. In addition to the genes for ammonium and nitrite oxidation, a complete urea degradation pathway was also retrieved in the genomes of comammox Nitrospira, ${ }^{5}$ yet the role of urea in their metabolism and its influence on their enrichment is still not clear.

Indeed, comammox Nitrospira has been widely detected in various natural and engineered systems, such as drinking water production and distribution systems, ${ }^{10,11,12,13}$ agricultural soils, ${ }^{14,15}$ and wastewater treatment processes. ${ }^{16-18}$ In these aforementioned investigations, comammox Nitrospira nearly always cooccur with other nitrifiers, with whom they compete for the same substrates (mainly ammonia and oxygen). As mentioned above, their kinetic properties indicate that highly oligotrophic habitats and systems favoring slow growth are preferred niches facilitating the dominance of comammox Nitrospira. ${ }^{8}$ However, to date there are only very few enriched comammox Nitrospira cultures, and only one pure culture available, ${ }^{5,6,8}$ all of which were obtained after intensive cultivation efforts. Therefore, we asked what specific niche would be favorable for comammox Nitrospira? To answer this question, we monitored the nitrifying community in a membrane bioreactor (MBR) fed with real urine wastewater. It appeared that the slow ammonia release from urea hydrolysis favored the enrichment of comammox Nitrospira in less than 200 days.

\footnotetext{
${ }^{1}$ School of Environment, Tsinghua University, Beijing, China. ${ }^{2}$ Department of Environmental Science and Engineering, University of Science and Technology of China, Hefei, People's Republic of China. ${ }^{3}$ Advanced Water Management Centre, Faculty of Engineering, Architecture and Information Technology, The University of Queensland, Brisbane, QLD, Australia. ${ }^{4}$ Department of Microbiology, IWWR, Radboud University, Nijmegen, AJ, The Netherlands. ${ }^{5}$ These authors contributed equally: Jiyun Li, Zheng-Shuang Hua. 然mail: wangcw@tsinghua.edu.cn; m.zheng@awmc.uq.edu.au; j.guo@awmc.uq.edu.au
}

Received: 28 January 2021 Revised: 4 February 2021 Accepted: 8 February 2021

Published online: 25 March 2021 


\section{MATERIALS AND METHODS}

\section{Bioreactor operation and sampling}

A continuous-flow MBR made from Plexiglass with a working volume of $12 \mathrm{~L}$ was used for enrichment (Supplementary Fig. S1). The reactor was installed with a submerged hollow fiber ultrafiltration membrane module ( $0.02 \mu \mathrm{m}$ pore size, Litree, China) with a total membrane surface area of $0.03 \mathrm{~m}^{2}$. A level control system was set up to prevent liquid overflowing. The reactor was fed with diluted real urine with Total Kjeldahl Nitrogen (TKN) concentration of $140-405 \mathrm{mg} \mathrm{N} \mathrm{L}^{-1}$ (for detailed influent composition see Supplementary Table S1). Initially, the reactor was inoculated with activated sludge taken from the aeration tank of a municipal wastewater treatment plant (Tsinghua Campus Water Reuse). The $\mathrm{pH}$ was maintained at $6.0 \pm 0.1$ by adding $1 \mathrm{M} \mathrm{NaOH}$ to buffer acidification by ammonia oxidation. The airflow was controlled at $2 \mathrm{~L} \mathrm{~min}^{-1}$, leading to the dissolved oxygen (DO) concentration above $4 \mathrm{mg} \mathrm{O}_{2} \mathrm{~L}^{-1}$ as regularly measured by a DO probe (WTW Multi 3420). The airflow also served to wash the membrane and mix the liquid. The temperature was controlled at $22-25^{\circ} \mathrm{C}$. The initial hydraulic retention time (HRT) was 3 days and was decreased to 1.5 days on day 222. The sludge retention time (SRT) was infinite as no biomass was discharged.

The MBR was operated for 490 days. During this period, influent and effluent samples ( $10 \mathrm{~mL}$ each) were collected 1-3 times per week and used to determine the concentrations of TKN, total nitrite nitrogen (TNN = $\mathrm{NO}_{2}{ }^{-} \mathrm{N}+\mathrm{HNO}_{2}-\mathrm{N}$ ), and nitrate nitrogen, according to standard methods. ${ }^{19}$ Mixed liquid samples $(25 \mathrm{~mL})$ were also taken weekly to measure mixed-liquor suspended solids (MLSS) and mixed-liquor volatile suspended solids (MLVSS). ${ }^{19}$ Biomass samples $(10 \mathrm{~mL}$ ) were regularly taken for qPCR and microbial community analyses (see below).

\section{Batch tests}

In order to test urea hydrolysis and subsequent nitrification in the enrichment culture, short-term incubations were performed in a cylindrical batch reactor $(8 \times 18.5 \mathrm{~cm}[\mathrm{~d} \times \mathrm{h}]$, made from Plexiglass). $150 \mathrm{~mL}$ biomass was sampled from the reactor and washed three times in $1 \times$ PBS buffer to remove any remaining nitrogen source. Subsequently, the biomass was resuspended in a $400 \mathrm{~mL}$ growth medium, which contained urea (about $\left.40 \mathrm{mg} \mathrm{N} \mathrm{L}^{-1}\right), \mathrm{NaHCO}_{3}\left(120 \mathrm{mg} \mathrm{L}^{-1}\right)$, and $2 \mathrm{~mL}$ Hunter's trace elements stock. Dissolved oxygen was controlled above $4 \mathrm{mg} \mathrm{O}_{2} \mathrm{~L}^{-1}$. Biotic and abiotic controls were performed under identical conditions with $\mathrm{NH}_{4} \mathrm{Cl}$ $\left(\sim 40 \mathrm{mg} \mathrm{N} \mathrm{L}^{-1}\right)$ instead of urea. The $\mathrm{pH}$ in all batch assays was maintained at $6.0 \pm 0.1$ by adding $1 \mathrm{M} \mathrm{HCl}$ or $\mathrm{NaOH}$. According to the microbial activities during long-term operation, each batch assay lasted 6 to $8 \mathrm{~h}$, and samples $(5 \mathrm{~mL})$ were taken every 20 to $60 \mathrm{~min}$. Biomass was removed by sterile syringe filter $(0.45 \mu \mathrm{m}$ pore size, JINTENG, China), and urea, ammonium, nitrite, and nitrate concentrations were determined as described above. All experiments were performed in triplicate.

\section{DNA extraction}

Biomass $(2 \mathrm{~mL})$ for DNA extraction was collected on days $0,53,98,131$, $161,189,210,238,266,301,321,358,378,449$, and 471. DNA was extracted using the FastDNA ${ }^{\text {TM }}$ SPIN Kit for Soil (MP Biomedicals, CA, U.S.) according to the manufacturer's protocols. DNA purity and concentration were examined using agarose gel electrophoresis and spectrophotometrically on a NanoDrop 2000 (ThermoFisher Scientific, Waltham, MA, USA).

\section{S rRNA gene amplicon sequencing and data analysis}

The V4-V5 region of the $16 \mathrm{~S}$ rRNA gene was amplified using the universal primers 515F (5'-barcode-GTGCCAGCMGCCGCGG-3') and $907 \mathrm{R}$ (5'CCGTCAATTCMTTTRAGTTI- $\left.3^{\prime}\right) .{ }^{20}$ PCR products were purified using the AxyPrep DNA Gel Extraction Kit (Axygen Biosciences, Union City, CA, USA) according to manufacturer's instructions and quantified using the QuantiFluor $^{\mathrm{TM}}$-ST (Promega, USA). Amplicons were pooled in equimolar concentrations and sequenced using the Illumina MiSeq PE3000 sequencer as per the manufacturer's protocol. Amplicon sequences were demultiplexed and quality filtered using QIIME (version 1.9.1). ${ }^{21}$ Reads $<50$ bp were discarded and all remaining paired-end reads with an overlap $>10$ bp were assembled. UPARSE (version 7.0.1090 http://drive5.com/uparse/) was used to cluster operational units (OTUs) on a $97 \%$ similarity cut-off level, and UCHIME to identify and remove chimeric sequences. The taxonomy of each 16S rRNA gene sequence was assigned by the RDP Classifier algorithm (http://rdp.cme.msu.edu/) according to the SILVA (SSU132) 16S rRNA database using a confidence threshold of $70 \%$.

\section{Quantification of various amoA by qPCR}

To quantify the abundances of comammox Nitrospira, $\mathrm{AOB}$ and $\mathrm{AOA}$ in the bioreactor, $\mathrm{qPCR}$ targeting the functional marker gene $a m o A$ was performed on DNA extracted from the bioreactor at different time points. We used the specific primers Ntsp-amoA 162F/359R amplifying comammox Nitrospira clades A and clade B simultaneously, ${ }^{12}$ Arch-amoAF/amoAR targeting AOA $a m o A,{ }^{22}$ and amoA-1F/amoA-2R for AOB amoA. ${ }^{23}$ Reactions were conducted on a Bori 9600plus fluorescence quantitative $P C R$ instrument using previously reported thermal profiles (Supplementary Table S2). Triplicate PCR assays were performed the appropriately diluted samples $\left(10-30 \mathrm{ng} \mathrm{LL}^{-1}\right)$ and 10-fold serially diluted plasmid standards as described by Guo et al. ${ }^{24}$. Plasmid standards containing the different $a m o A$ variants were obtained by TA-cloning with subsequent plasmid DNA extraction using the Easy Pure Plasmid MiniPrep Kit (TransGen Biotech, China). Standard curves covered three to eight orders of magnitude with $R^{2}$ greater than 0.999 . The efficiency of qPCR was about $95 \%$.

\section{Library construction and metagenomic sequencing}

The extracted DNA was fragmented to an average size of about $400 \mathrm{bp}$ using Covaris M220 (Gene Company Limited, China) for paired-end library construction. A paired-end library was constructed using NEXTFLEX Rapid DNA-Seq (Bioo Scientific, Austin, TX, USA). Adapters containing the full complement of sequencing primer hybridization sites were ligated to the blunt-end of fragments. Paired-end sequencing was performed on lllumina NovaSeq PE150 (Illumina Inc., San Diego, CA, USA) at Majorbio Bio-Pharm Technology Co., Ltd. (Shanghai, China) using NovaSeq Reagent Kits according to the manufacturer's instructions (www.illumina.com).

\section{Metagenomic assembly and genome binning}

Raw metagenomic sequencing reads (in PE150 mode) were trimmed and quality filtered with in-house Perl scripts as described previously. ${ }^{25}$ Briefly, duplicated reads caused by the PCR bias during the amplification step were dereplicated. Reads were eliminated if both paired-end reads contained $>10 \%$ ambiguous bases (that is, " $\mathrm{N}$ "). Low-quality bases with phred values $<20$ at both sides were trimmed. This resulted in on average 57 million high-quality pair-ended reads for each dataset. Quality-filtered reads from each sample were assembled separately using SPAdes v3.9.0 ${ }^{26}$ with the following parameters: -meta $-\mathrm{k} 33,55,77,99,127$. Contigs $>2.5 \mathrm{kbp}$ were retained for later analysis. Genome binning was conducted for each sample using sequencing depth and tetranucleotide frequency. To calculate coverage, high-quality reads from all samples were mapped to the contigs using BBMap v38.85 (http://sourceforge.net/projects/bbmap/) with minimal identity set to $90 \%$. The generated bam files were sorted using samtools v1.3.1. ${ }^{27}$ Then, sequencing depth was calculated using the script "jgi_summarize_bam_contig_depths" in MetaBAT. ${ }^{28}$ Metagenomeassembled genomes (MAGs) were obtained in MetaBAT. MAG quality, including completeness, contamination, and heterogeneity, was estimated using CheckM v1.0.12. ${ }^{29}$ To optimize the MAGs, emergent self-organizing maps $^{30}$ were used to visualize the bins, and contigs with abnormal coverage or discordant tetranucleotide frequencies were removed manually. Finally, all MAGs were reassembled using SPAdes with the following parameters: -careful $-k 21,33,55,77,99,127$. The reads used for reassembly were recruited by mapping all high-quality reads to each MAG using BBMap with the same parameter settings as described above.

\section{Functional annotation of metagenomic assemblies and metagenome-assembled genomes}

Gene calling was conducted for the complete metagenomic assemblies and all retrieved MAGs using Prodigal v2.6.3.31 For the MAGs, predicted protein-coding sequences (CDSs) were subsequently aligned to a manually curated database containing $a m o C A B$, hao, and $n \times r A B$ genes collected from public database using DIAMOND v0.7.9 (E-values $<1 \mathrm{e}-5^{32}$ ) MAGs found to contain all these genes were labeled as comammox Nitrospira MAGs and kept for later analysis. Functional annotations were obtained by searching all CDSs in the complete metagenomic assemblies and the retrieved MAGs against the NCBI-nr, eggNOG, and KEGG databases using DIAMOND $(E$-values $<1 \mathrm{e}-5)$.

\section{Phylogenetic analysis}

Phylogenomic tree. The taxonomic assignment of the three identified comammox Nitrospira MAGs was determined using GTDB-tk v0.2.2. ${ }^{33}$ To 
a
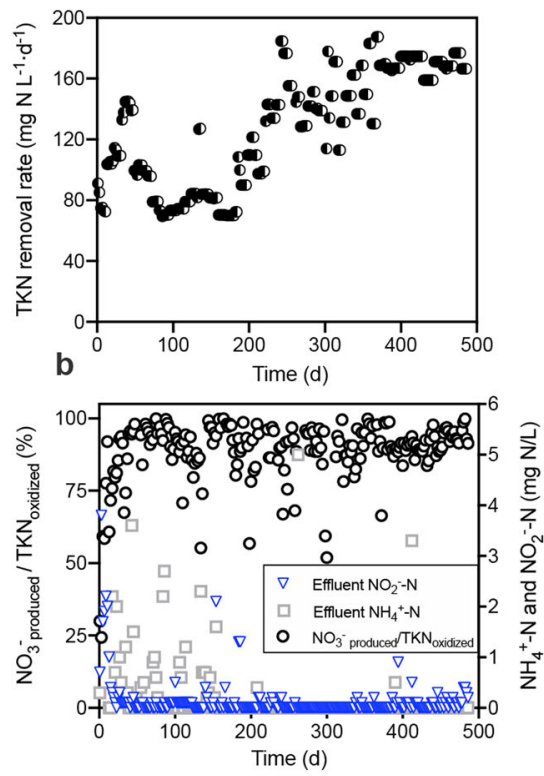

C
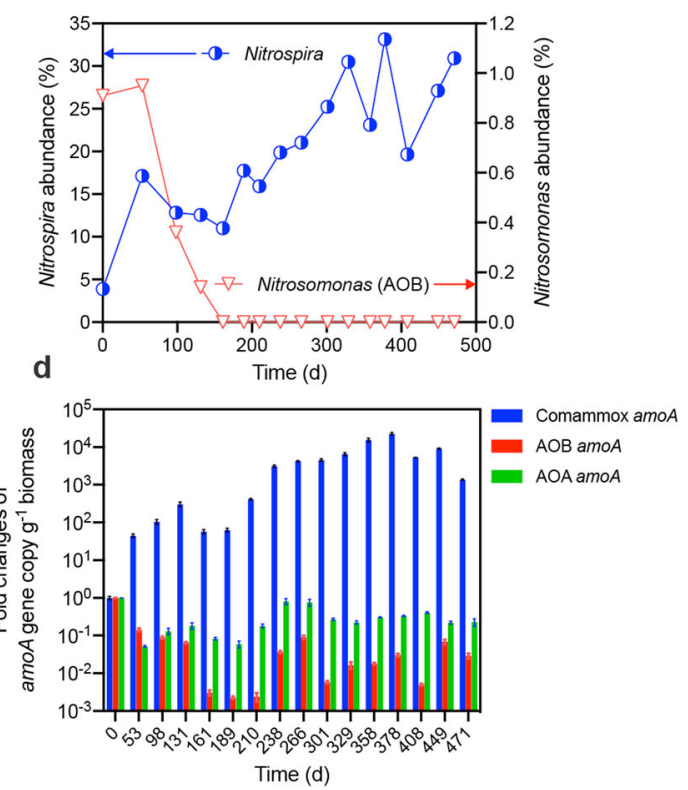

Fig. 1 Nitrification performance and nitrifying community composition of the urine-fed MBR. a TKN removal rate. $\mathbf{b}$ Ammonium and nitrite conversion. c Variations in the relative abundance of Nitrospira and Nitrosomonas based on 16S rRNA gene amplicon sequencing. d qPCRbased quantification of comammox Nitrospira, AOB, and AOA amoA genes.

reveal the phylogenetic placement of these MAGs within the Nitrospirae, 296 genomes from this phylum were downloaded from the NCBI-RefSeq database. The download genomes were dereplicated using $\mathrm{dRep} v 2.3 .2^{34}$ (-con 10 -comp 80 ) to reduce the complexity and redundancy of the phylogenetic tree, which resulted in the removal of 166 genomes. In the remaining genomes, the three comammox Nitrospira MAGs and 25 genomes from phylum Thermotogae which were treated as outgroups, a set of 16 ribosomal proteins were identified using AMPHORA2. ${ }^{35}$ Each gene set was aligned separately using MUSCLE v3.8.31 with default parameters, ${ }^{36}$ and poorly aligned regions were filtered by TrimAl v1.4.rev22 (-gt 0.95 -cons $50^{37}$ ) The individual alignments of the 16 marker genes were concatenated, resulting in an alignment containing 118 species and 2665 amino acid positions. Subsequently, the best phylogenetic model $L G+F+R 8$ was determined using ModelFinder ${ }^{38}$ integrated into IQ-tree v1.6.10. ${ }^{39}$ Finally, a phylogenetic tree was reconstructed using IQ-tree with the following options: -bb 1000 -alrt 1000. The generated tree in newick format was visualized by iTOL v3. ${ }^{40}$

amoA tree. Reference $a m o A$ sequences of $A O B, A O A$, and comammox Nitrospira were obtained from NCBI. Together with the amoA genes from the present study, all sequences were aligned and trimmed as described above. IQ-tree was used to generate the phylogenetic tree, with "LG + G4" determined as the best model.

ure $A B C$ gene tree. ure $A B C$ gene sequences detected in this study were extracted and used to build a database using "hmmbuild" command in HMMER $^{41}$ ureABC gene sequences from genomes in NCBI-RefSeq database (downloaded on July 1st, 2019) were identified by searching against the built database using AMPHORA2. The same procedures as above were conducted to construct the phylogenetic tree of concatenated ureABC genes, except for the sequence collection step. To reduce the complexity of the phylogenetic tree, the alignment of concatenated ure $A B C$ genes was clustered using CD-HIT ${ }^{42}$ with the following parameters: - $\mathrm{aS} 1-\mathrm{c} 0.8-\mathrm{g} 1$. Only representative sequences were kept for phylogeny reconstruction, which resulted in an alignment containing 858 sequences and 1263 amino acids positions. "LG + R10" was determined as the best model and used to build the phylogenetic tree. Regarding the Nitrospirae-specific ureABC gene tree, ure $A B C$ gene sequences were recruited from the genomes as described above, but without the sequence clustering step. The final Nitrospirae-specific phylogeny of ure $A B C$ genes was built on an alignment containing 62 sequences and 1015 amino acid positions with "LG $+F+$ $\mathrm{I}+\mathrm{G} 4^{\prime \prime}$ as the best model.

\section{RESULTS}

Selective enrichment of comammox Nitrospira in urine-fed MBR

Initially, an MBR with a working volume of $12 \mathrm{~L}$ (Supplementary Fig. S1) was inoculated with nitrifying activated sludge from a municipal wastewater treatment plant with the aim to treat source-separated urine. The reactor was fed with real urine wastewater (for detailed influent compositions see Supplementary Table S1) for 490 days in total without intentional biomass removal (corresponding to an infinite SRT). Unlike any of the previous enrichments of comammox Nitrospira, ${ }^{5,6}$ oxygen in this reactor was supplied in excess to maintain the DO concentration above $4 \mathrm{mg} \mathrm{O}_{2} \mathrm{~L}^{-1}$. As expected, stable nitrification performance was attained rapidly at a controlled $\mathrm{pH}$ of $6.0 \pm 0.1$, in terms of ammonium conversion to nitrate, with negligible nitrite accumulation $\left(0.1 \pm 0.4 \mathrm{mg} \mathrm{NO}_{2}^{-}-\mathrm{N} \mathrm{L}^{-1}\right)$. Upon a subsequent gradual decrease in HRT, the MBR achieved a maximum TKN removal rate of $188 \mathrm{mg} \mathrm{N} \mathrm{L}^{-1} \mathrm{~d}^{-1}$ (Fig. 1a). The ratio of $\mathrm{NO}_{3}{ }^{-}$produced to $\mathrm{TKN}_{\text {oxidized }}$ was nearly $100 \%$ (Fig. 1b). Batch incubations with urea $\left(39 \pm 0.3 \mathrm{mg} \mathrm{N} \mathrm{L}^{-1}\right)$ as the only nitrogen source were performed to simulate urine conversion to nitrate and confirm bona fide urea degradation (Supplementary Fig. S2). While we observed simultaneous urea degradation and nitrate increase, some ammonium still accumulated $\left(8.4 \pm 0.9 \mathrm{mg} \mathrm{N} \mathrm{L}^{-1}\right)$ in the first $3.5 \mathrm{~h}$, but subsequently decreased to $0 \mathrm{mg} \mathrm{N} \mathrm{L}^{-1}$ within the next $2.5 \mathrm{~h}$ (Supplementary Fig. S2a). While the urea degradation seems to be independent of ammonium oxidation (Supplementary Fig. S2c), there was no urea hydrolysis observed in the abiotic control (Supplementary Fig. S2d). These results suggest that the microbial community present in the MBR could use urea as an alternative ammonium source, which was converted into nitrate.

Biomass was periodically sampled for $16 \mathrm{~S}$ rRNA gene amplicon sequencing (Supplementary Fig. S3). Surprisingly, Nitrosomonas was the only detected canonical AOB with a relative abundance of $0.91 \%$ in the inoculum (day 0) but became undetectable from day 161 onwards (Fig. 1c). As no AOA-affiliated genus or NOB other than Nitrospira were detected in the enriched culture, we probed for the presence of comammox Nitrospira by using quantitative PCR (qPCR; Fig. $1 \mathrm{~d}$ and Supplementary Table S2). During the long-term operation, 
the copy number of comammox Nitrospira amoA genes increased up to $10^{4}$ times, whereas a decreasing trend was observed for $A O B$ amo $A$ and $A O A$ amoA genes if compared with the inoculum. After 300 days of enrichment, Nitrospira represented up to $33 \%$ of the total $16 \mathrm{~S}$ rRNA genes in the population based on amplicon sequencing (Supplementary Fig. S3). These results collectively indicated that comammox Nitrospira had been selectively enriched in this urine-fed MBR in less than 200 days, while AOB and AOA seemed to play only a minor role in the system.

\section{Metagenomic sequencing and binning}

The genus Nitrospira consists of at least six distinct sublineages, with all known comammox organisms affiliated with sublineage II. However, within this sublineage, the comammox Nitrospira does not form a monophyletic clade and cannot reliably be recognized based on 16S rRNA gene amplicon sequencing. ${ }^{10}$ Thus, to further characterize the microbial community, we sequenced the complete metagenome of three samples (collected on day 189, 266, and 378; see "Materials and methods" section), which allowed the reconstruction of three high-quality MAGs belonging to Nitrospira (Supplementary Fig. S4 and Supplementary Table S3).

To identify the potential for complete nitrification in the recovered Nitrospira, the predicted CDSs in each MAG were probed for the presence of amoCAB, hao, and nxrAB, encoding the functional subunits of ammonia monooxygenase, hydroxylamine dehydrogenase, and nitrite oxidoreductase, respectively. Indeed, the complete suite of functional genes for ammonia oxidation ( $a m o C A B$ and $h a o$ ) were identified in all three MAGs, together with the genes for nitrite oxidation (nxrAB), thus confirming that these MAGs belong to comammox Nitrospira. The observed GC content, genome size, number of protein-coding genes, and the coding density are all in the range of previously published comammox Nitrospira genomes (Supplementary Table S3). ${ }^{5,14,43,44}$ Estimated completeness and contamination of the three Nitrospira MAGs were $>90 \%$ and $<3 \%$, respectively, indicating their high quality and good resolution.

\section{Identification of three new comammox Nitrospira}

Phylogenetic analysis using a concatenated alignment of 16 conserved single-copy bacterial marker genes showed the placement of three recovered comammox Nitrospira MAGs within comammox Nitrospira clade A of Nitrospira sublineage II (Fig. 2a), which also includes the genomes of all previously enriched comammox Nitrospira. ${ }^{5,6,9}$ Read mapping against the assembled metagenomes indicated that the microbial community in the MBR at all sampling time points was dominated by comammox Nitrospira, with total relative abundances of up to $30 \%$ (Fig. 2b), which is in good agreement with the total Nitrospira with $\leq 33 \%$ observed by amplicon sequencing. In addition, the relative abundance of the only canonical nitrite-oxidizing Nitrospira MAG (identified based on metagenomics, Supplementary Table S4) gradually decreased from $4.0 \%$ (day 189) to $1.8 \%$ (day 378 ). Together with the results of $16 \mathrm{~S}$ rRNA gene amplicon sequencing and $\mathrm{qPCR}$, this further substantiates the dominance of comammox Nitrospira in the MBR. Comparative genomic analyses revealed that the three comammox Nitrospira MAGs had average amino acid identities (AAl) $\leq 77 \%$ with 25 publicly available genomes of comammox Nitrospira and $\leq 86 \%$ with each other (Fig. 2c), indicating that they represent new species as these are well below the $95 \%$ AAl cut-off proposed for species delineation. ${ }^{45}$ This evolutionary divergence was also underpinned by the phylogenomic distances to their closest relatives (Fig. 2c). Consistently, the $a m o A$ sequences of the three comammox Nitrospira MAGs obtained in this study clustered within comammox Nitrospira clade A, confirming their affiliation with this distinct group (Supplementary Fig. S5)

\section{Urea degradation by comammox Nitrospira}

To infer the potential impact of urea degradation during the enrichment, the urea metabolism in the three MAGs was analyzed in detail. The full genetic complement for urea utilization was indeed present in all three species (Fig. 3a, b). The ureABC core genes were localized on a contig along with urease accessory genes (ureDFGH). Furthermore, genes for an $A B C$-type urea transporter (urt $A B C D E$ ) were identified directly upstream of ure $A B C$. While genes responsible for urea utilization were also detected in other community members, including bacteria affiliated with Rhodospirillales, Rhizobiales, and Actinobacteria (Supplementary Fig. S6), the comammox Nitrospira were most likely the key contributors for urea degradation in this system. This is underlined by the high relative abundance of comammox Nitrospira-derived ureC accounting for $41-66 \%$ of all ureC genes based on metagenomic read mapping. Moreover, the existence of ureC in other Nitrospirae indicated that canonical NOB might also contribute to urea degradation at a certain level. Remarkably, phylogenetic analysis showed that the UreABC protein sequences from the phylum Nitrospirae form a distinct deeply branching cluster within the protein family (Fig. 3c), indicating urea hydrolysis being an ancient trait of the genus Nitrospira. Together with the absence of sequences from non-Nitrospirae species within this sequence group, the monophyly of this lineage strongly suggests vertical inheritance of this trait from their last common ancestor. The observation that most non-Nitrospira members in this phylum are not possessing urease is most likely explained by frequent gene loss within these lineages. Within the genus Nitrospira, the ureABC of the three comammox Nitrospira obtained in this study form a monophyletic cluster, together with ureases of other clade A affiliates (Fig. 3d), which is in line with concatenated marker gene and amoAbased results. To identify the respective contribution of canonical NOB and comammox Nitrospira to urea degradation, further investigation of ureC expression of each genome is needed.

\section{DISCUSSION}

Until now, only a few comammox Nitrospira have successfully been enriched, as it is not yet clear how to promote their growth over other nitrifiers besides applying substrate-limited conditions favoring slow growth. ${ }^{8}$ Indeed, the previously obtained enrichments of comammox Nitrospira were initiated from biofilms in engineered systems, which fulfilled the conditions of long SRT and low substrate concentrations., ${ }^{5,6}$ Furthermore, low oxygen levels were recognized as a critical factor in the competition between comammox Nitrospira and other nitrifiers in natural and manmade systems. ${ }^{46,47}$ However, in this study, the enrichment of comammox Nitrospira was achieved at DO concentrations above $4 \mathrm{mg} \mathrm{O}_{2} \mathrm{~L}^{-1}$, which indicates that oxygen may not be a decisive factor to favor comammox Nitrospira over other nitrifiers. This observation is in line with a recent study surveying twelve wastewater treatment plants, which also showed no obvious correlation between oxygen levels and abundances of comammox Nitrospira. ${ }^{17}$ Below we discuss the potential factors that could instead have been responsible for the selective enrichment of comammox Nitrospira in $<200$ days in the present study.

Firstly, the reactor was fed in continuous mode at low pH of $6.0 \pm$ 0.1 , leading to an extremely low free ammonia concentration of $\sim 30 \mathrm{nM}$. Meanwhile, besides biomass loss during samplings, there was no sludge discharging in the MBR, causing an infinite SRT. Presumably, both low ammonia availability and long SRT contributed to the enrichment of comammox Nitrospira. However, there are more natural systems with such conditions that still are dominated by other nitrifiers. For example, acidic forest soils, in which ammonia concentrations were also negligible at a $\mathrm{pH}$ below 5.0 and SRT can be considered as long as hundreds of days, showed one to two orders of magnitude higher amoA-based abundances of AOA than comammox Nitrospira. ${ }^{10,48}$ These observations indicate that long SRT and low ammonia availability are not the only decisive factors for the dominance of comammox Nitrospira.

Notably, one of the critical features of the present study was urea supply in the reactor medium, which likely was another 


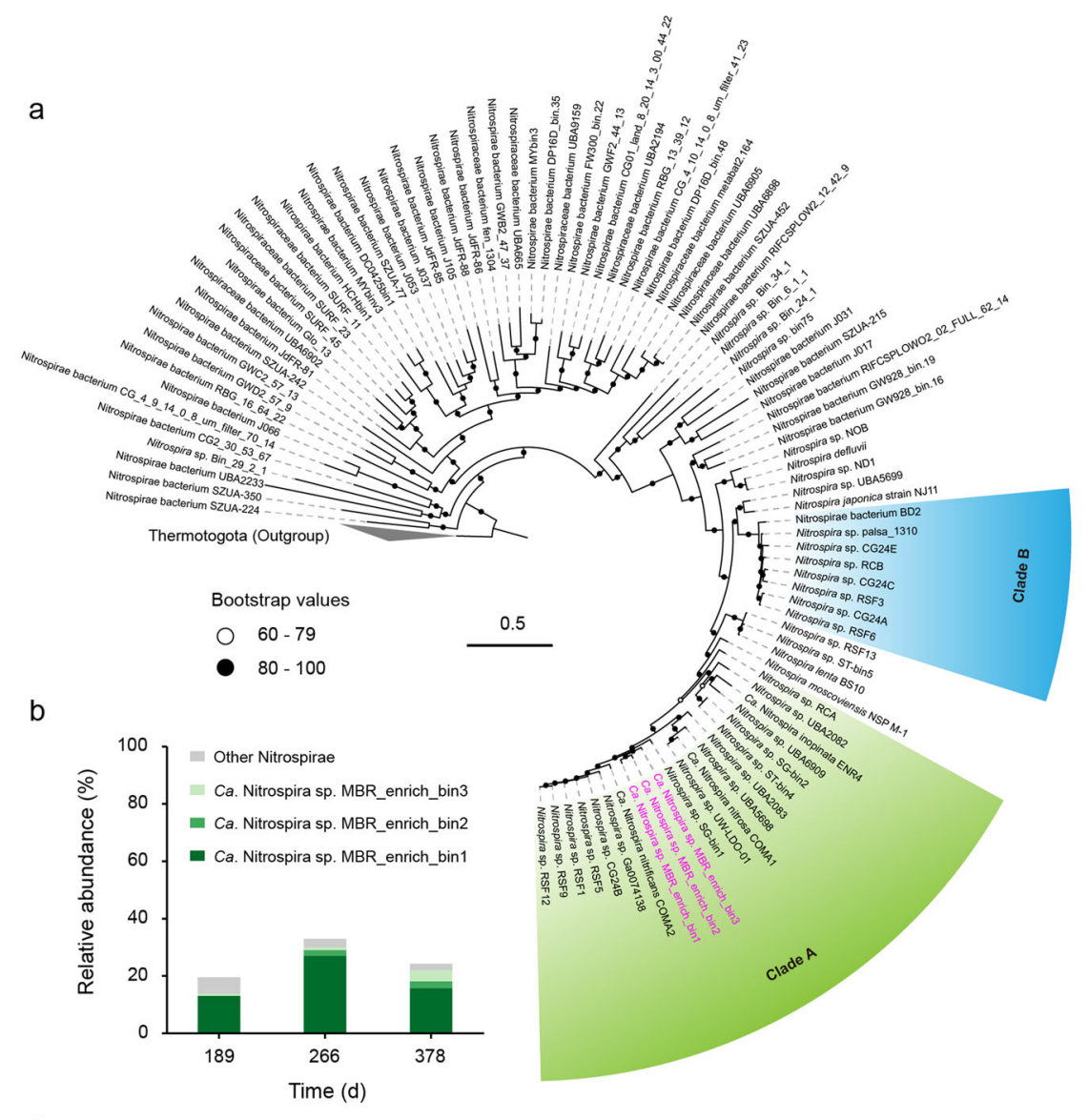

C
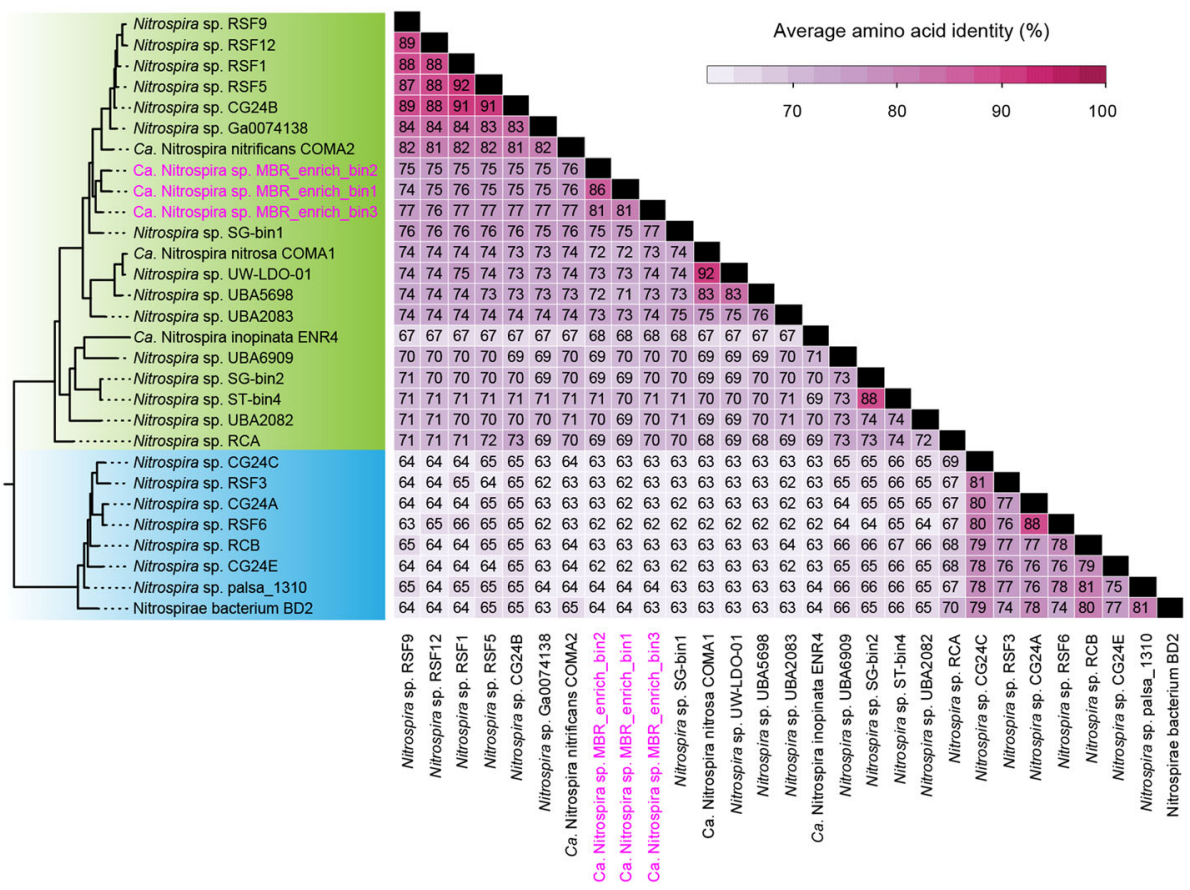

Fig. 2 Phylogenetic affiliation and abundance of the three novel comammox Nitrospira. a Maximum-likelihood phylogenetic tree constructed on the basis of 16 concatenated ribosomal proteins. Reference genomes were downloaded from the NCBI-RefSeq database. Genomes from Thermotogae are used as outgroups. Comammox Nitrospira clade A and B Nitrospira are indicated by green and blue shading, respectively. $\mathbf{b}$ Relative abundances of comammox Nitrospira bins recovered from the individual metagenomes. The relative abundances were calculated as proportions of recruited clean reads mapped to a specific MAG to all clean reads. c Average amino acid identities (AAls) calculated for comammox Nitrospira clade A and B genomes. The sub-tree on the left was extracted from the phylogenetic tree in a. Heatmap on the right gives the average AAl values between all 28 comammox Nitrospira genomes. Genomes retrieved in this study are shown in pink in a and $\mathbf{c}$. 
a

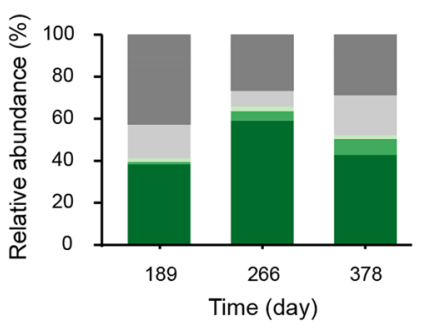

c
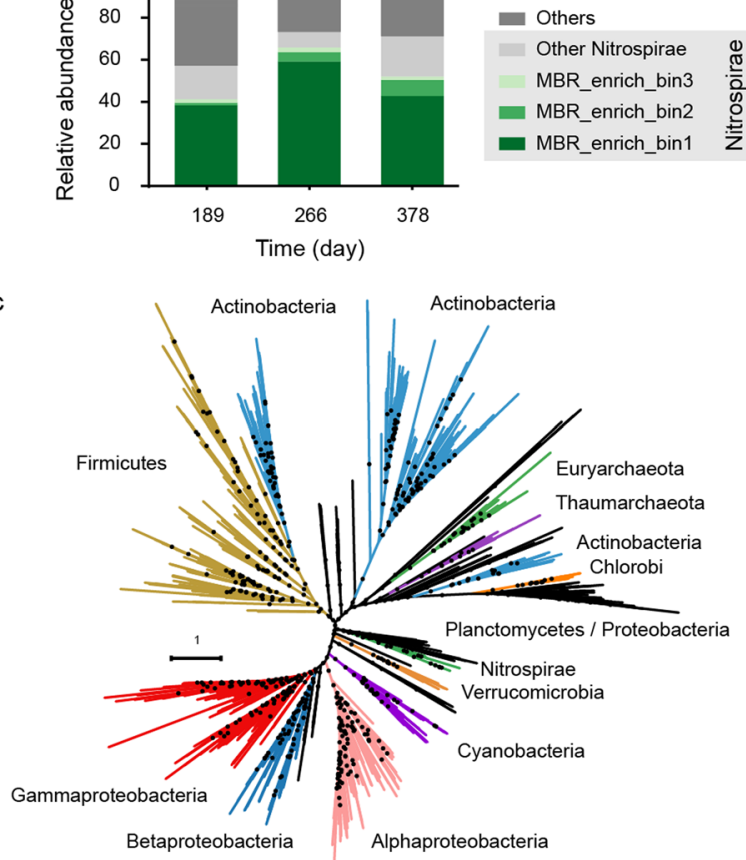

b
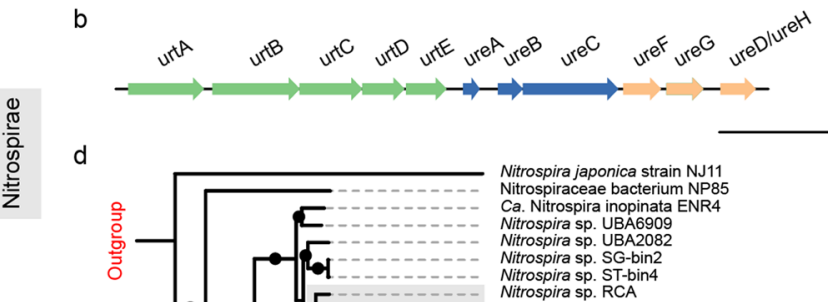

d

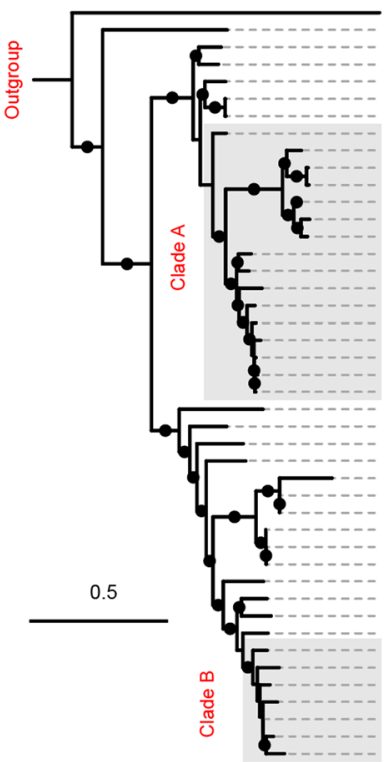

Nitrospira japonica strain NJ11 Nitrospiraceae bacterium NP85
Ca. Nitrospira inopinata ENR4 Ca. Nitrospira inopinata
Nitrospira sp. UBA6909 Nitrospiria sp. UBA2082 Nitrospira sp. SG-bin2 Nitrospira sp. ST-bind Nitrospira sp. RCA Ca. Nitrospira nitrosa COMA1 Nitrospira sp. UW-LDO-01 Ca. Nitrospira sp. MBR_enrich_bin3 1BR-enrich bin 1 Nitrospira sp. SG-bin1 1 Nitrospira sp. UBA2083
Nitrospira Sp. UBA5698 Nitrospira sp. UBA5. RSF12 Nitrospira sp. RSF9
Nitrospira s. G0074138 Nitrospira sp. RSF1 Nitrospira Sp. CG24B Nitrospira sp. RSF5 Nitrospira sp. bin75 Nitrospipirae bacterium GW928 bin 19 UMm RIFCSPLOWO2 NODE 6039 length 9910 cov 4.017888 NODE 25911 length 24391 cov 3.817837 NODE 932 length 57785 cov 12.718218 NODE 543 length 57785 cov 15.91267 ODE 543 length 59387 cov 19.71103 Nitrospira sp. ND1 1 Nitrospira moscoviensis NSP M-1 Nitrospira sp. $\mathrm{BSA}$.
Nitrospira Nitrospira lenta BS10 10 Nitrospira sp. CG24E Nitrospira sp. RSF6 Nitrospira sp. CG24A Nitrospira sp. CG24C

Fig. 3 Detected ure operons in MBR community members. a Relative abundance of urease-containing microorganisms in the community. All ureC genes were extracted from the three metagenomes and their relative abundances were estimated by calculating the proportions of mapped reads from a specific ureC gene to all detected ureC genes. $\mathbf{b}$ Schematic representation showing the arrangement of the urease and urea transporter operons in Nitrospira. Arrows represent genes and indicate the transcriptional direction. Maximum-likelihood-based phylogeny of concatenated protein sequences of ure $A B C$ genes extracted from c all publicly available prokaryotic genomes and $\mathbf{d}$ all Nitrospira genomes. Both trees were calculated with 1000 bootstrap replications, bootstrap support $\geq 70 \%$ is indicated by black circles.

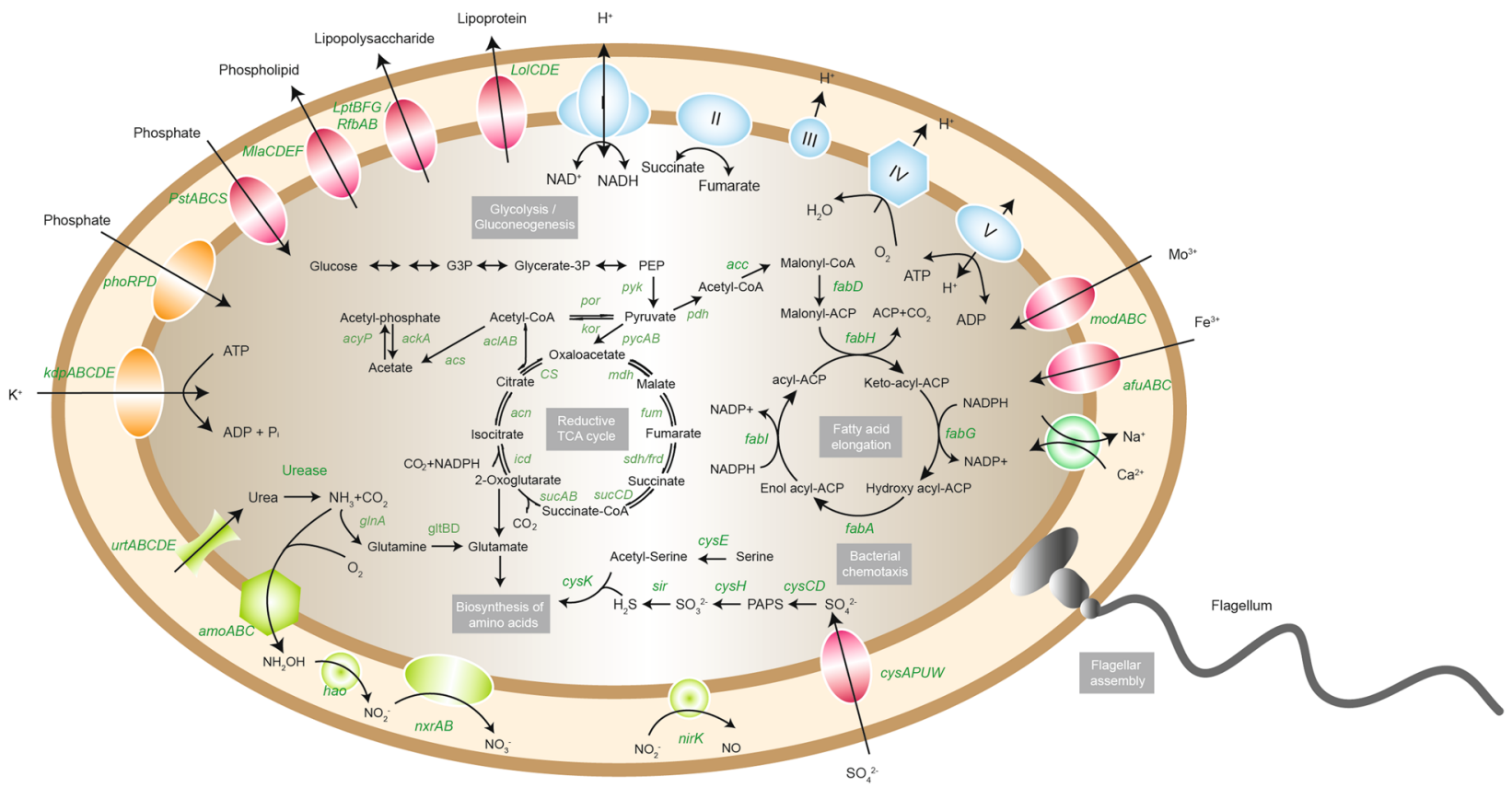

Fig. 4 Cell metabolic diagram constructed from the MBR comammox Nitrospira genome annotations. Only genes relevant for the carbon, nitrogen, and energy metabolisms, and nutrient transport are shown. Solid lines indicate genes detected in the genomes of comammox Nitrospira retrieved in this study.

contributor for selectively enriching comammox Nitrospira. A complete urea utilization pathway was recovered from the enriched comammox Nitrospira, which agreed with previous studies. ${ }^{5,43,44}$ Due to the presence of the nickel-dependent urease (UreABC), accessory proteins (UreDFG), and ATP-dependent ABC- type urea transporter (UrtABCDE), urea can be enzymatically hydrolyzed to ammonia and $\mathrm{CO}_{2}$ by comammox Nitrospira (Fig. 4). Besides its role in assimilation and amino acid biosynthesis, the produced ammonia can fuel nitrification. Notably, although a small fraction of $A O B$ and $A O A$ can also use urea as energy and 
nitrogen source under ammonium-limited conditions, ${ }^{49-52}$ the ureolytic capability is recognized as a general feature for comammox Nitrospira, as the relevant genes (ureABC) have been detected in almost every genome of comammox Nitrospira retrieved up to date. ${ }^{18}$ Moreover, different kinetics for urea likely contributed to the selective success of comammox Nitrospira as well. For example, if the affinity constant of urea for comammox Nitrospira is indeed lower than other urea-utilizing microorganisms, they could be selectively enriched under urea-limiting conditions. This hypothesis requires experimental investigation in the future.

Urea, an organic nitrogen compound ubiquitously present in marine and freshwater systems, agricultural soil, and domestic sewage, is derived from both natural and anthropogenic sources. ${ }^{53}$ Although the utilization of urea has been identified in various microbial groups, the enrichment of novel comammox Nitrospira on this substrate is remarkable and adds to their important role in the microbial nitrogen cycle. Generally, ammonia oxidation is considered as the initial rate-limiting step of nitrification, also causing close cooperation between $A O B / A O A$ and NOB. Recently, it has been proposed that ureolytic NOB can also initiate nitrification by cleaving urea into ammonia and $\mathrm{CO}_{2}$ which will then reciprocally feed the AOB/AOA. ${ }^{7,54}$ Evidenced by the fact that the majority of ureC was derived from the retrieved comammox Nitrospira genomes and the successful enrichment of comammox Nitrospira in a short time, the present study further supports that a single microorganism can carry out all reactions to convert urea to nitrate on its own, ${ }^{7,49-52}$ and also highlighted that urea could be a potential selective factor for the enrichment of comammox Nitrospira. Further investigation by culture-dependent experiments will provide more solid evidence.

\section{DATA AVAILABILITY}

All data supporting the findings of this study are available in this paper and the Supplementary Information. Sequencing data are deposited at the NCBI under the project of PRJNA637405, with raw sequence data under accession numbers SRR11928969-SRR11928971, and the draft genomes of three new comammox Nitrospira under the Biosample accession numbers SAMN15105088-SAMN15105090.

\section{REFERENCES}

1. Winogradsky, S. Recherches sur les organismes de la nitrification. Ann. Inst. Pasteur. 4, 11-12 (1890)

2. Könneke, M. et al. Isolation of an autotrophic ammonia-oxidizing marine archaeon. Nature. 437, 543-546 (2005).

3. Treusch, A. H. et al. Novel genes for nitrite reductase and Amo-related proteins indicate a role of uncultivated mesophilic crenarchaeota in nitrogen cycling. Environ. Microbiol. 7, 1985-1995 (2005).

4. Costa, E., Pérez, J. \& Kreft, J.-U. Why is metabolic labour divided in nitrification? Trends Microbiol. 14, 213-219 (2006).

5. van Kessel, M. A. et al. Complete nitrification by a single microorganism. Nature 528, 555-559 (2015).

6. Daims, H. et al. Complete nitrification by Nitrospira bacteria. Nature 528, 504 (2015).

7. Koch, $\mathrm{H}$. et al. Expanded metabolic versatility of ubiquitous nitrite-oxidizing bacteria from the genus Nitrospira. Proc. Natl Acad. Sci. USA 112, 11371-11376 (2015).

8. Kits, K. D. et al. Kinetic analysis of a complete nitrifier reveals an oligotrophic lifestyle. Nature 549, 269-272 (2017).

9. Sakoula, D. et al. Enrichment and physiological characterization of a novel comammox Nitrospira indicates ammonium inhibition of complete nitrification. ISME J. 1-15 (2020).

10. Pjevac, P. et al. AmoA-targeted polymerase chain reaction primers for the specific detection and quantification of comammox Nitrospira in the environment. Front. Microbiol. 8, 1508 (2017).

11. Pinto, A. J. et al. Metagenomic evidence for the presence of comammox Nitrospira-Like bacteria in a drinking water system. mSphere. 1, e00054-15 (2016).

12. Fowler, S. J. et al. Comammox Nitrospira are abundant ammonia oxidizers in diverse groundwater-fed rapid sand filter communities. Environ Microbiol. 20, 1002-1015 (2018).
13. Palomo, A. et al. Metagenomic analysis of rapid gravity sand filter microbial communities suggests novel physiology of Nitrospira spp. ISME J. 10, 2569-2581 (2016).

14. Orellana, L. H. et al. Year-round shotgun metagenomes reveal stable microbial communities in agricultural soils and novel ammonia oxidizers responding to fertilization. Appl. Environ. Microbiol. 84, e01646-01617 (2018).

15. Xu, S. et al. Ubiquity, diversity, and activity of comammox Nitrospira in agricultural soils. Sci. Total Environ. 706, 135684 (2020).

16. Bartelme, R. P., McLellan, S. L. \& Newton, R. J. Freshwater recirculating aquaculture system operations drive biofilter bacterial community shifts around a stable nitrifying consortium of ammonia-oxidizing archaea and comammox Nitrospira. Front. Microbiol. 8, 101 (2017).

17. Cotto, I. et al. Long solids retention times and attached growth phase favor prevalence of comammox bacteria in nitrogen removal systems. Water Res. 169, 115268 (2020).

18. Spasov, E. et al. High functional diversity among Nitrospira populations that dominate rotating biological contactor microbial communities in a municipal wastewater treatment plant. ISME J. 14, 1857-1872 (2020).

19. APHA. Standard Methods for the Examination of Water and Wastewater (2005).

20. Stubner, S. Enumeration of 16S rDNA of Desulfotomaculum lineage 1 in rice field soil by real-time PCR with SybrGreen ${ }^{\text {TM }}$ detection. J. Microbiol. Meth. 50, 155-164 (2002).

21. Caporaso, J. G. et al. QIIME allows analysis of high-throughput community sequencing data. Nature methods. 7, 335 (2010).

22. Francis, C. A. et al. Ubiquity and diversity of ammonia-oxidizing archaea in water columns and sediments of the ocean. Proc. Natl Acad. Sci. USA 102, 14683-14688 (2005).

23. Rotthauwe, J.-H., Witzel, K.-P. \& Liesack, W. The ammonia monooxygenase structural gene amoA as a functional marker: molecular fine-scale analysis of natural ammonia-oxidizing populations. Appl. Environ. Microbiol. 63, 4704-4712 (1997).

24. Guo, J. et al. Metagenomic analysis of anammox communities in three different microbial aggregates. Environ Microbiol. 18, 2979-2993 (2016).

25. Hua, Z. S. et al. Ecological roles of dominant and rare prokaryotes in acid mine drainage revealed by metagenomics and metatranscriptomics. ISME J. 9, 1280-1294 (2015).

26. Bankevich, A. et al. SPAdes: a new genome assembly algorithm and its applications to single-cell sequencing. J. Comput. Biol. 19, 455-477 (2012).

27. Li, H. et al. The sequence Alignment/Map format and SAMtools. Bioinformatics. 25, 2078-2079 (2009).

28. Kang, D. D., Froula, J., Egan, R. \& Wang, Z. MetaBAT, an efficient tool for accurately reconstructing single genomes from complex microbial communities. PeerJ. 3, e1165 (2015).

29. Parks, D. H. et al. CheckM: assessing the quality of microbial genomes recovered from isolates, single cells, and metagenomes. Genome Res. 25, 1043-1055 (2015).

30. Dick, G. J. et al. Community-wide analysis of microbial genome sequence signatures. Genome Biol. 10, R85 (2009).

31. Hyatt, D. et al. Prodigal: prokaryotic gene recognition and translation initiation site identification. BMC Bioinformatics. 11, 119 (2010)

32. Buchfink, B., Xie, C. \& Huson, D. H. Fast and sensitive protein alignment using DIAMOND. Nat. Methods. 12, 59-60 (2015).

33. Chaumeil P. A., Mussig A. J., Hugenholtz P. \& Parks D. H. GTDB-Tk: a toolkit to classify genomes with the genome taxonomy database. Bioinformatics. 11, 119 (2019).

34. Olm, M. R., Brown, C. T., Brooks, B. \& Banfield, J. F. dRep: a tool for fast and accurate genomic comparisons that enables improved genome recovery from metagenomes through de-replication. ISME J. 11, 2864-2868 (2017).

35. Wu, M. \& Scott, A. J. Phylogenomic analysis of bacterial and archaeal sequences with AMPHORA2. Bioinformatics. 28, 1033-1034 (2012).

36. Edgar, R. C. MUSCLE: multiple sequence alignment with high accuracy and high throughput. Nucleic Acids Res. 32, 1792-1797 (2004).

37. Capella-Gutierrez, S., Silla-Martinez, J. M. \& Gabaldon, T. trimAl: a tool for automated alignment trimming in large-scale phylogenetic analyses. Bioinformatics. 25, 1972-1973 (2009).

38. Kalyaanamoorthy, S. et al. ModelFinder: fast model selection for accurate phylogenetic estimates. Nat Methods. 14, 587-589 (2017).

39. Nguyen, L. T., Schmidt, H. A., von Haeseler, A. \& Minh, B. Q. IQ-TREE: a fast and effective stochastic algorithm for estimating maximum-likelihood phylogenies. Mol. Biol. Evol. 32, 268-274 (2015).

40. Letunic, I. \& Bork, P. Interactive tree of life (iTOL) v3: an online tool for the display and annotation of phylogenetic and other trees. Nucleic Acids Res. 44, W242-W245 (2016).

41. Johnson, L. S., Eddy, S. R. \& Portugaly, E. Hidden Markov model speed heuristic and iterative HMM search procedure. BMC Bioinformatics. 11, 431 (2010).

42. Li, W. \& Godzik, A. Cd-hit: a fast program for clustering and comparing large sets of protein or nucleotide sequences. Bioinformatics. 22, 1658-1659 (2006). 
43. Poghosyan, L. et al. Metagenomic recovery of two distinct comammox Nitrospira from the terrestrial subsurface. Environ. Microbiol. 21, 3627-3637 (2019).

44. Palomo, A. et al. Comparative genomics sheds light on niche differentiation and the evolutionary history of comammox Nitrospira. ISME J. 12, 1779-1793 (2018).

45. Luo, C., Rodriguez-r, L. M. \& Konstantinidis, K. T. MyTaxa: an advanced taxonomic classifier for genomic and metagenomic sequences. Nucleic Acids Res. 42, e73-e73 (2014).

46. Keene-Beach, N. \& Noguera, D. R. Design and assessment of species-level qPCR primers targeting comammox. Front. Microbiol. 10, 36 (2019).

47. Roots, P. et al. Comammox Nitrospira are the dominant ammonia oxidizers in a mainstream low dissolved oxygen nitrification reactor. Water Res. 157, 396-405 (2019).

48. Shi, X. et al. Niche separation of comammox Nitrospira and canonical ammonia oxidizers in an acidic subtropical forest soil under long-term nitrogen deposition. Soil Biol. Biochem. 126, 114-122 (2018).

49. Kitzinger, K. et al. Cyanate and urea are substrates for nitrification by Thaumarchaeota in the marine environment. Nat. Microbiol. 4, 234-243 (2019).

50. Alonso-Sáez, L. et al. Role for urea in nitrification by polar marine Archaea. Proc. Natl Acad. Sci. USA 109, 17989-17994 (2012).

51. Tourna, M. et al. Nitrososphaera viennensis, an ammonia oxidizing archaeon from soil. Proc. Natl Acad. Sci. USA 108, 8420-8425 (2011).

52. Pommerening-Röser, A. \& Koops, H.-P. Environmental $\mathrm{pH}$ as an important factor for the distribution of urease positive ammonia-oxidizing bacteria. Microbiol. Res. 160, 27-35 (2005)

53. Solomon, C. M., Collier, J. L., Berg, G. M. \& Glibert, P. M. Role of urea in microbial metabolism in aquatic systems: a biochemical and molecular review. Aquat. Microb. Ecol. 59, 67-88 (2010).

54. Daims, H., Lücker, S. \& Wagner, M. A new perspective on microbes formerly known as nitrite-oxidizing bacteria. Trends Microbiol. 24, 699-712 (2016).

\section{ACKNOWLEDGEMENTS}

This work was financially supported by the National Natural Science Foundation of China (21876095). J.G. acknowledges the Australian Research Council for funding support through Australian Research Council Future Fellowship (FT170100196). M.Z. acknowledges the support of an Early Career Researcher Award at The University of Queensland. M.S.M.J. is supported by the Netherlands Organisation for Scientific Research (NWO; Gravitation Grant SIAM 024.002.002) and the European Research Council (ERC Synergy Grant Marix 854088). S.L. is supported by NWO (grant 016. Vidi.189.050).

\section{AUTHOR CONTRIBUTIONS}

J.G., C.W. and M.Z. conceived and designed this study. J.L., C.W. and G.B. operated reactors, performed $\mathrm{qPCR}$ and analytical measurements, and prepared biomass for $16 \mathrm{~S}$ rRNA gene amplicon sequencing and metagenomics. Z.-S.H., J.L., J.G. and S.L. performed metagenomic analyses. T.L., C.W. and M.Z. performed data analyses of reactor performance and $16 \mathrm{~S}$ rRNA gene amplicon sequencing. JG., M.Z., M.S.M.J. and S.L. provided critical biological interpretations of the data. T.L. wrote the paper with input from all authors.

\section{COMPETING INTERESTS}

The authors declare no competing interests.

\section{ADDITIONAL INFORMATION}

Supplementary information The online version contains supplementary material available at https://doi.org/10.1038/s43705-021-00005-3.

Correspondence and requests for materials should be addressed to C.W., M.Z. or J.G.

Reprints and permission information is available at http://www.nature.com/ reprints

Publisher's note Springer Nature remains neutral with regard to jurisdictional claims in published maps and institutional affiliations.

(i) Open Access This article is licensed under a Creative Commons C. Attribution 4.0 International License, which permits use, sharing, adaptation, distribution and reproduction in any medium or format, as long as you give appropriate credit to the original author(s) and the source, provide a link to the Creative Commons license, and indicate if changes were made. The images or other third party material in this article are included in the article's Creative Commons license, unless indicated otherwise in a credit line to the material. If material is not included in the article's Creative Commons license and your intended use is not permitted by statutory regulation or exceeds the permitted use, you will need to obtain permission directly from the copyright holder. To view a copy of this license, visit http://creativecommons. org/licenses/by/4.0/. 